\title{
Correction to: A numerical scheme to solve a class of two-dimensional nonlinear time-fractional diffusion equations of distributed order
}

\author{
A. Babaei ${ }^{1} \cdot$ H. Jafari ${ }^{1,2,3} \cdot$ S. Banihashemi ${ }^{1}$
}

Published online: 19 February 2021

๑) Springer-Verlag London Ltd., part of Springer Nature 2021

Correction to: Engineering with Computers

https://doi.org/10.1007/s00366-020-01185-7

In the original publication of the article, the corresponding author has been incorrectly tagged as A. Babaei. The correct corresponding author should be H. Jafari, email ID: jafarh@ unisa.ac.za.

The original article has been corrected.

H. Jafari

jafarh@unisa.ac.za

A. Babaei

babaei@umz.ac.ir

1 Department of Applied Mathematics, University of Mazandaran, Babolsar, Iran

2 Department of Mathematical Sciences, University of South Africa, UNISA0003, Pretoria, South Africa

3 Department of Medical Research, China Medical University Hospital, China Medical University, Taichung 110122,

Taiwan 